Meta

Journal des tradlucteurs

Translators' Journal

\title{
Le point de vue de l'éditeur
}

\section{Pierre Tisseyre}

Volume 14, numéro 1, mars 1969

URI : https://id.erudit.org/iderudit/004492ar

DOI : https://doi.org/10.7202/004492ar

Aller au sommaire du numéro

Éditeur(s)

Les Presses de l'Université de Montréal

ISSN

0026-0452 (imprimé)

1492-1421 (numérique)

Découvrir la revue

Citer cet article

Tisseyre, P. (1969). Le point de vue de l'éditeur. Meta, 14(1), 31-33.

https://doi.org/10.7202/004492ar

Ce document est protégé par la loi sur le droit d'auteur. L’utilisation des services d'Érudit (y compris la reproduction) est assujettie à sa politique d'utilisation que vous pouvez consulter en ligne.

https://apropos.erudit.org/fr/usagers/politique-dutilisation/
Cet article est diffusé et préservé par Érudit.

Érudit est un consortium interuniversitaire sans but lucratif composé de l’Université de Montréal, l'Université Laval et l'Université du Québec à Montréal. Il a pour mission la promotion et la valorisation de la recherche. https://www.erudit.org/fr/ 


\section{DStrons}

\section{le point de vue de l'éditeur}

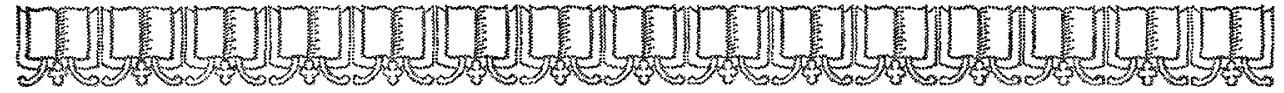

Pour un éditeur, la publication d'un ouvrage traduit pose tout d'abord un problème matériel. En effet, il faut rémunérer le traducteur et dans un pays comme le nôtre où le marché est de peu d'importance, et par conséquent, les droits d'auteur modestes, on trouve peu de traducteurs de valeur qui acceptent d'être payés en partageant les droits d'auteur avec l'auteur original.

Cette formule du partage des droits d'auteur qui est de règle au théâtre, par exemple, n'existe pour le livre que dans les grands pays et même là, elle se limite à la traduction d'ouvrages célèbres dont la vente s'annonce considérable. Dans tous les autres cas, le traducteur est rémunéré par un paiement forfaitaire. Cela veut dire que l'éditeur doit débourser en plus des frais normaux de publication, c'est-à-dire ceux qui résultent de la composition, de l'impression, du lancement et de la publicité, une somme importante qu'il ne peut espérer récupérer qu'en augmentant le prix du livre, ce qui a pour effet de diminuer ses chances de succès, ou en sollicitant et obtenant une subvention.

Le Conseil des arts du Canada, depuis plus de dix ans, s'intéresse particulièrement à la traduction dans l'une ou l'autre des deux langues officielles du pays. Pour un ouvrage de valeur qui paraît chez nous en français, un éditeur de Toronto obtient assez facilement une subvention pour défrayer le coût de sa traduction en anglais. De même, nous rencontrons peu de difficultés pour obtenir une subvention lorsqu'il s'agit de traduire des ouvrages importants parus en langue anglaise.

Malheureusement, la subvention est rarement suffisante pour payer la totalité du montant demandé par un bon traducteur et l'éditeur doit acquitter la différence. De plus — et c'est là un élément non négligeable - , une partie de notre public, on pourrait presque dire la meilleure, étant bilingue, préfère prendre connaissance d'une œuvre littéraire dans sa langue originale, ce qui diminue automatiquement le marché déjà restreint.

Ces considérations commerciales étaient nécessaires avant d'aborder les autres aspects de la question, car elles conditionnent en grande partie l'édition d'ouvrages 
traduits. Pour ceux que cet aspect matérialiste du problème laisse froids, j'ajouterai cependant qu'aussi longtemps que l'édition restera confiée à l'entreprise privée, sa rentabilité est une condition essentielle de son existence. Il ne faut donc pas s'étonner du peu d'ouvrages traduits qui paraissent au Québec.

$\mathrm{Si}$ on aborde les problèmes de la qualité en traduction, on s'aperçoit que là encore, contrairement à ce qu'on pourrait imaginer, on se heurte à de graves difficultés.

On pourrait croire que chez nous, où la connaissance de l'anglais est si répandue, les traducteurs abondent. Pour les mauvais traducteurs, c'est exact; pour les bons, c'est faux.

Qu'est-ce en effet qu'un bon traducteur? C'est d'abord et avant tout un écrivain qui devrait posséder un talent au moins égal à celui de l'auteur étranger qu'il traduit. Quand il a plus de talent que lui c'est encore mieux et l'exemple qui vient immédiatement à l'esprit, c'est celui de Baudelaire, traducteur d'Edgar Allan Poe. Or, lorsque nous avons la chance de posséder un écrivain authen'ique, nous attendons de lui des cuvres originales et non pas qu'il mette son talent au service d'auteurs étrangers. Il lui faudrait d'ailleurs beaucoup d'abnégation pour accepter de rester dans l'ombre d'un autre quand il y a tant de place à prendre au soleil chez nous.

Ceci étant acquis, il faut également qu'un traducteur ait une très bonne connaissance de la langue dans laquelle écrit l'auteur qu'il veut traduire. Cette remarque a l'air d'être une lapalissade, mais elle est nécessaire parce que le bilinguisme dont se réclament un grand nombre de nos intellectuels, ne correspond pas à une connaissance profonde de l'anglais. Nous apprenons tous l'anglais par l'oreille et par la vue puisque nous vivons sur un ilôt qu'assaille une mer anglosaxonne, mais cette connaissance qui suffit largement aux besoins quotidiens et qui peut, dans une conversation, faire illusion à des Anglais eux-mêmes, ne s'appuie pas sur les infrastructures que donnent de solides études.

Disons-le, souvent quelqu'un se croit traducteur alors qu'il lui manque la culture profonde dans les deux langues qui est indispensable - tout au moins lorsqu'il s'agit de traduction littéraire. Je laisse à des linguistes le soin de développer ce point, même si ces derniers ont parfois tendance à donner la première p'ace aux spécialistes de la linguistique alors qu'à mon avis, le meilleur traducteur, à talent égal, ne sera pas le meilleur linguiste des deux, mais celui dont la culture dans les deux langues sera la plus profonde.

Dans le domaine du manuel scolaire où les problèmes matériels que j’ai mentionnés ci-dessus ne jouent plus puisqu'il s'agit d'un marché qui permet, en cas de succès, de réaliser de gros tirages, il est encore plus difficile de trouver un bon traducteur. En effet, aux qualités indispensables mentionnées ci-dessus, il faut en ajouter d'autres tout aussi importantes: une expérience pédagogique et des connaissances dans la discipline particulière du manuel que l'on veut traduire.

Le traducteur idéal d'un manuel de mathémathiques, par exemple, devrait être un bon professeur de mathématiques. Or, là encore, chez nous, on se heurte, surtout dans le domaine scientifique, à une difficulté particulière.

Je ne voudrais offenser personne, mais je suis souvent consterné lorsque j'écoute des professeurs qui à la télévision viennent discuter avec une grande 
compétence des problèmes de leur spécialité. Souvent, trop souvent, ils s'expriment dans une langue effroyable, déformée par les anglicismes. La raison, tout le monde la connaît, puisque, à une époque très récente, il fallait aller parfaire ses études aux États-Unis et que, même encore aujourd'hui, on fait des études supérieures en utilisant des manuels de langue anglaise. C'est donc aller très souvent à une catastrophe que de confier la traduction d'un manuel scolaire à l'un des plus éminents spécialistes de sa discipline.

Pour remédier à cette situation, on a proposé d'adjoindre à ces spécialistes un linguiste qui serait chargé de remettre en bon français le premier jet du traducteur. Mais une telle dualité ne va pas sans heurts!

Les spécialistes tiennent en effet dur comme fer à leur terminologie, même si elle constitue un jargon, et cela pour une raison bien simple, c'est que l'esprit préfère utiliser, pour raisonner, un outil imparfait qu'il connaît bien à un outil parfait mais inconnu.

Le linguiste, lui, ne s'intéresse qu'à la langue, au point même de se désintéresser des nécessités pédagogiques. Il pourchassera par exemple l'anglicisme au point de reconstruire des expressions usuelles, claires et foncièrement correctes parce que, par hasard, elles sont calquées sur la phrase anglaise correspondante. Entre le spécialiste furieux qu'on "abîme » son texte et le linguiste prêt à défendre la langue française jusqu'à la mort, l'éditeur un beau jour se voit forcé d'être arbitre car il y a des impératifs de date de parution qui exigent de mettre fin à ce genre de discussion.

Un mot, pour terminer, du coût des traductions. Il est trop élevé. Les traducteurs professionnels ont été amenés à établir un tarif au mot qui se comprend parfaitement puisque $90 \%$ des traductions « anglais-français », «français-anglais » qui se font dans le Québec sont des textes commerciaux: annonces, communiqués, circulaires, etc. De là à vouloir appliquer ce tarif au mot lorsqu'il s'agit d'un livre, il y a un abîme.

Aussi longtemps que ce problème ne sera pas résolu, on continuera pour traduire des livres à se passer des services de traducteurs professionnels, en faveur d'amateurs qui, s'ils sont consciencieux, passeront sur les pages d'un livre quatre ou cinq fois plus de temps qu'il n'en faudrait à un traducteur professionnel.

$\mathrm{Si}$ j'ai énuméré les difficultés, c'est parce qu'elles me paraissent d'une extrême gravité et qu'il semble que bien souvent on les méconnaisse. Elles sont difficiles à surmonter mais on y parvient, puisque nous avons vu récemment un écrivain de la qualité de Jean Simard prêter son talent à Hugh MacLennan et que d'excellentes traductions de manuels scolaires ont été réalisées dernièrement.

Il y a cependant du chemin à faire pour que chez nous le mot traduction n'ait pas un sens péjoratif et qu'on puisse l'employer sans qu'inconsciemment votre interlocuteur le fasse précéder de l'adjectif «mauvais ».

Pierre Tisseyre 